Journal of Social Sciences 1 (4): 199-202, 2005

ISSN 1549-3652

(c) 2005 Science Publications

\title{
Using Neutral Network in Predicting Corporate Failure
}

\author{
Huong Giang Nguyen \\ School of Banking and Finance, The University of New South Wales \\ Sydney - New South Wales - Australia
}

\begin{abstract}
This study investigates the predictive power of three neutral network models: Multi-layer neural network, probabilistic neural network, and logistic regression model in predicting corporate failure. Basing on the database provided by The Corporate Scorecard Group (CSG), we combine financial ratios which deem to be significant predictors of corporate bankruptcy in many previous empirical studies to build our predictive models and test it against the holdout sample. On comparison of the results, we find that three models are good at predicting probability of corporate failure. Moreover, probabilistic neural network model outperforms the others. Therefore, neutral networks are useful and probabilistic neutral network is a promising tool for the prediction of corporate failure.
\end{abstract}

Key words: Corporate failure, default risk, credit risk, neutral network

\section{INTRODUCTION}

When examining the bankruptcy costs, Warner ${ }^{[1]}$ proposes that controlling the number of firms that fail is important in order to guarantee sustainable economic growth. Therefore, it will be of great value if a financial model that can predict corporate failure with a high degree of certainty can be produced.

The development of corporate failure prediction models started in America in the mid-1960 and there have been a large number of studies on this subject since then. These studies are conducted firstly within the boundary of the parametric statistical analysis such as dicriminant or logit functions. However, it is argued that traditional statistical models usually assume multivariate normality and homoscedastic variances, and these assumptions are often violated in real world financial data ${ }^{[2]}$.

Recent developments in the field of non-parametric statistical analysis establishes neutral networks as an efficient approach in identifying complicated relationships in multidimensional datasets, without making a priori assumptions regarding the nature of these relationships.

The comparison the neutral networks and traditional statistic models in predicting corporate failure have been analyzed by many researchers so far. Some suggest that they should be used as a complementary $^{[3]}$ while the others suggest that neutral networks work better than disciminant analysis ${ }^{[4]}$.

This study will look deeply at the neutral networks. Using the Corporate Scorecard Group's (CSG) databases and the ANGOSS Knowledge Studio software, we investigate three neutral networks models: probabilistic neural network, multilayer neural network and logistic regression in predicting corporate failure in Australia in period 1988- 2002.
Economists have developed two approaches for analysis of corporate failure: the traditional statistical approach i.e., linear discriminant analyses, and an artificial intelligence algorithm known as neural networks. Amongst the earliest studies from the traditional statistic approach are the ones of Beveaver and Altman who concentrated on predicting business failure of US companies.

Beaver ${ }^{[5]}$ finds various financial ratios could be useful predictors of corporate failure even five years before the event. In particular, the author shows that profitability ratios, liquidity ratios and solvency ratios appeared to be the most significant predictors of corporate failure. However, ratio analysis can be misinterpreted and ambiguous. Because for most ratios, there is no benchmark to compare against and it is subject to the manager's choice whether a particular ratio is satisfactory.

Altman introduces well known models in predicting corporate failure. Z-Score model is introduced in 1968, Zeta model in 1977 and these models are re-discussed again in 2000. He discusses multiple discriminant analysis, which is a statistical technique that categorizes an observation into predetermined groups based on the observations of individual characteristics. Multiple disciminant analysis is advantageous over ratio analysis because it is able to simultaneously analyze all the characteristics of the variable by considering all the characteristics common to the relevant firms, as well as looking at the interactions between these characteristics.

However, traditional statistical models usually assume multivariate normality and homoscedastic variances, and these assumptions are often violated in real world financial data ${ }^{[2]}$. Therefore, it is proposed that neutral networks would be an alternative approach

Corresponding Author: Huong Giang Nguyen, School of Banking and Finance, The University of New South Wales, Sydney - New South Wales - Australia 
to the traditional approach and many studies have been conducted on comparing these two approaches.

Altman et $a .^{[3]}$ compares between traditional statistical methodologies for distress classification and prediction, i.e., linear discriminant or logit analyses, with neural networks. By analyzing well over 1,000 healthy, vulnerable and unsound industrial Italian firms from 1982-1992, their results show that there is a balanced degree of accuracy and other beneficial characteristics between linear discriminant analyses and neutral networks. They are particularly careful to point out the problems of the 'black-box' neutral network systems, including illogical weightings of the indicators and overlitting in the training stage both of which negatively impacts predictive accuracy. They conclude that there certainly should be further studies and tests using the two techniques and suggest a combined approach for predictive reinforcement.

Ragothaman $^{[2]}$ uses financial statement data from COMPUSTAT to build a neural network model, and test it against a holdout sample, and against two statistical models - a linear model and a non-linear one as well. A set of training examples comprising $75 \mathrm{CIB}$ and 75 NCIB firms are used to develop the neural network model, and a separate holdout sample containing $25 \mathrm{CIB}$ and $25 \mathrm{NCIB}$ firms is used to validate the eight-variable neural network predictions. The performance of the neural network is also compared to that of a multiple discriminant analysis model and a logistic regression model, using the same data. Results indicate that the neural network model predicts CIB case better than the two statistical models and the statistical models outperform the neutral network model in predicting the NCIB and hence neural network and Logit/MDA models can be considered complementary to one another, not necessarily alternatives.

Zapranis and Ginoglou ${ }^{[4]}$ contrast the neutral networks approach with multivariable discriminate analysis in predicting corporate failure in Greece. Their results indicate that neutral networks outperform the linear approach, within sample average classification rate of 95 percent. The equivalent in-sample figure for multivariate discriminant analysis was 85 percent. The increased classification rates of neutral networks can be attributed to their improved ability to classify the problematic firms.

Yim and Mitchell ${ }^{[6]}$ investigate whether two artificial neural networks (ANNs), multilayer perceptron (MLP) and hybrid networks using statistical and ANN approaches, can outperform traditional statistical models for predicting corporate failures in Australia one year and two years prior to the financial distress during period 1995 and 1999. The results suggest that hybrid neural networks outperform all other models one and two years before failure.

This study will look deeply at the neutral network models in testing the case of Australia with supporting of ANGOSS Knowledge Studio software. We use financial data provided by CSG to analyze the predictive power of neural network models: The MultiLayer Neural Network, The Probabilistic Neural Network, and the Logistic Regression Model.

\section{DATA DESCRIPTION}

Two sets of data provided the Corporate Scorecard Group's (CSG) will be used for our analysis. The first dataset consists of 32 Australian companies which is called the training or sample dataset. These companies are known in terms of their financial information and de-listed or failed status between 1988 and 2002. Data used for the failed firm is from the last and penultimate financial statement issued before the firm failed. Thus, the prediction of failure is to be made for up to two years in advance. The second dataset consists of 200 companies with the same financial information except that they are coded by the CSG and no extra defaulted information is revealed. Our aim is to predict the failed companies from this dataset by building and testing neutral network models, which will be done basing on the training of the sample data set.

Most failure prediction studies are interested in developing more accurate predictions by selecting the best financial ratios or the analysis. No unified theory has been recognized as a basis for theoretical ratio selection. We choose a set of financial ratios, which have successfully predicted firm failure in previous studies, to build the models including profitability, liquidity, solvency and financial leverage.

\section{NEUTRAL NETWORK PREDICTIVE MODELS}

Neutral networks are consistent paradigms of nonparametric approach in financial modeling. A neural network is made up of units that are based on the biological neuron. Each unit or neuron has a number of inputs that are combined to produce a single output. The output remains low until the combination of inputs reaches a threshold. Outputs from a layer of neurons are fed to another layer called the hidden layer. These outputs are then fed to a single neuron that produces the final output. Their major strength lies in the fact that they do not require any a priori assumptions regarding the underlying structure of the relationship they are estimating. Hence, neural networks have often been proposed as alternative solutions for business problems in a variety of situations ${ }^{[7]}$.

This study uses ANGOSS Knowledge Studio software to test the predictive power of three neutral network models: Probabilistic Neural Network, MultiLayer Neural Network, Logistic Regression. Predictive models are objects that are trained for the purpose of making predictions on new data based on patterns in existing data. There are three general stages: training, 
validation, and scoring. During the training phase, the model learns how to use some of the fields in a record to predict the value of another field. A patternrecognizing model that understands the data will be created. In the validation phase, the model is tested against another dataset and then verified as being reasonable. Pattern-recognizing models will be verified by making predictions on a dataset for which you already know the answers. In the scoring phase, the model is used to make a prediction on new datasets provided they have a structure similar to the one used for training.

Logistic Regression is a simple model where, for any given set of points, some line best fits the points. Logistic regression algorithm for models is used with a restricted range, binary or multi-nominal dependent variable. Multi-layer neural network is a network with one or more layers of hidden neurons. It can be used to train a multi-dimensional dataset with a continuous underlying model. Multi-layer neural network employs back propagation for training, which is slow to reach convergence and thus takes some time to train. Probabilistic neural network uses a memory based reasoning technique. It is considered a neural network on the basis that each training record is like a neuron. Creating a probabilistic neural network predictive model is actually very fast. The training set simply has to be normalized and copied. However, scoring can be quite time consuming as each record in the training set is visited ${ }^{[8]}$.

\section{EMPIRICAL INVESTIGATION}

Our process for developing predictive models starts with a search of the ASIC and the Delisted Company websites (www.delisted.com.au) to identify the companies that had actually faileded in the 32 training dataset. We identify 6 out of the 32 companies defined as bankrupt according to the criteria of being liquidated. That gives us the actual percentage of default companies in the training data set as $18.75 \%$.

Training process: We perform our analysis on three predictive models: Probabilistic Neural Network, Multilayer Neural Network, and Logistic Regression. We train the first dataset with various individual financial variables in order to identify a combination of sensitive variables which would give us the highest predictive accuracy of these models.

Validating process: If the training is satisfied with high accuracy, we then apply it to the validation. The result is shown in a matrix which compares the predictive result with the actual result in an effective way. Emphasis is placed on Type I and Type II errors, which we aim to keep as low as possible.
Scoring process: In this step, once we get a high accuracy level, we then apply the combination of variables derived from training dataset to the second dataset (200 companies), which we assume to have the same structure in term of size, industry, geographic location, etc, as the training dataset. Therefore, we expect the second dataset to have a similar percentage or proportion of failure as the training dataset. It means that around $18.75 \%$ of these companies would fall into default categories. We also use it as a benchmark when evaluating the performance of the three models.

Findings: For the Probabilistic neutral network, with the training process, the model correctly predicts 93.75\% both for one year and two years before failure with parameters includes: WC/Total Assets, RE/Total Assets, EBIT/Total Assets, Net sales/Total Assets. The model validation finds Type I and Type II errors of both 0 for one year before failure and 1 and 0 for the two years before failure. The scoring process shows that the model estimate the percentage of corporate failure is $18.5 \%$ (37 out of 200) and $22.55 \%$ (45 out of 200) for one and two years before failure respectively.

For the Multi-layer neural network, the predictive power of the model for one year before failure seems pretty good. During the training process, the model correctly predicts $95.45 \%$ with parameters including Sales/Total Assets, Current Liability Ratio, Sales/Fixed Assets, Interest Exp., OPBAT/Total Assets, Net Income/Net Sale. The model validation process finds Type I and Type II errors of both 0 . The scoring process shows that the model estimate the percentage of default is $15.5 \%$ ( 31 out of 200). However, for two year before failure, the model's predictive power seems extremely poor. Type II error during the validation process is 5 . It means that the model predicts 5 actual failed companies as non-failed one and the scoring process shows the percentage of failure is $0 \%$ (200 out of 200 companies are not failed).

For Logistic regression model, with the training process, the model correctly predicts $100 \%$ both for one year and two years before failure with parameters including Current Ratio, Quick Ratio, WC/Total Assets, Debt/Equity Ratio, EBIT/Total Assets, Sale/Total Capital, OPABT/Sale. The model validation finds Type I and Type II errors of both 0 for one year before and two years before failure. The scoring process shows that the model estimate the percentage of failure is very high with $38.5 \%$ (77 out of 200 ) and $44.5 \%$ (89 out of 200) for one and two years before failure respectively.

On comparison of three predictive models, different models use different sets of variables in order to get the highest level of predictive accuracy. Results from different models vary slightly as each model uses different mechanisms in discriminating the dummy variable. In all three predictive models, their predictive power is good with entropy explained of around $70 \%$ 
except for the prediction of multi-layer neutral network two years prior to failure. As moving close to the failure date, there are some improvements in the one year result over the two year result in terms of predictive power, Type I and Type II errors. The result also indicates that the Probabilistic Neural Network seems to perform better than the other models for following reasons: First, a pretty close percentage of failure in 200 dataset compared to the 32 training dataset $(18.75 \%$ ), which are 18.5 and $22.55 \%$ in one and two years before failure respectively. Second, consistent performance in both one year and two year dataset with no Type II error in either one and two year 32 companies dataset, no Type I error in one year before failure and only $3.57 \%$ (1 out of 27) Type I error two years before failure.

\section{CONCLUSION}

This study investigates the predictive power of three neutral network models: The Multi-Layer Neural Network, The Probabilistic Neural Network, and the Logistic Regression Model in predicting corporate failure both one year and two years before failure by testing the case of Australia. Using a set of sample data, various financial ratios have been selected to build the predictive models and the test the models on a larger dataset. The results shows that these models are good at predicting corporate failure and probabilistic neural network model outperforms the others due to its high accuracy levels using both the one year and two years before failure. Therefore, probabilistic neutral network is a promising tool for failure prediction in particular and hence this supports the conclusion that neutral networks would be useful for researchers, shareholders, policymakers and others interested in early warning system.

\section{ACKNOWLEDGEMENT}

The author is grateful for critical comments and helpful suggestions from Vic Eward, Yiping Rao, Vikki Wong, Bhakti Mittal, Ruibin Li. Any remaining errors are my own responsibilities.

\section{REFERENCES}

1. Warner, J.B., 1977. Bankruptcy Costs: Some Evidence. J. Finance, 17: 337-347.

2. Ragothaman, S., 2003. A neutral network approach to predicting corporate illegal behavior. J. Forensic Account., 4: 181-198.

3. Altman, E., 1994. Corporate distress diagnosis: comparisons using linear discriminant analysis and neural networks (the Italian experience). J. Banking and Finance, 18: 505-529.

4. Zapranis, A. and D. Ginoglou, 2000. Forecasting Corporate failure using neutral network approach: The Greece case. J. Financial Management and Analysis, 1: 11-20.

5. Beaver, W.H., 1967. Financial ratios as predictors of corporate failure. J. Account. Res., 5: 123-150.

6. Yim, J. and H. Mitchell, 2002. A comparison of corporate failure models in Australia: Hybrid neutral networks, logit models and discriminant analysis. RMIT Working Paper No.10/2002.

7. Trippi, R. and E. Turban, 1999. Neural Networks in Finance and Investing.

8. Christopher, M., 1995. Neural Networks for Pattern Recognition. 